\title{
Ecthyma gangrenosum as a serious complication of Pseudomonas aeruginosa infection in departments of paediatric oncology
}

\author{
Przemysław Gałązka1', Patryk Kaczor², Krystian Kałużny³, Kamil Leis²
}

${ }^{1}$ Department of General and Oncological Surgery for Children and Adolescents, Antoni Jurasz University Hospital No. 1 in Bydgoszcz, Ludwik Rydygier Collegium Medicum in Bydgoszcz, Nicolaus Copernicus University in Torun, Poland

${ }^{2}$ Faculty of Medicine, Ludwik Rydygier Collegium Medicum in Bydgoszcz, Nicolaus Copernicus University in Torun, Bydgoszcz, Poland ${ }^{3}$ Chair and Clinic of Rehabilitation, Antoni Jurasz University Hospital No. 1 in Bydgoszcz, Faculty of Health Sciences, Ludwik Rydygier Collegium Medicum in Bydgoszcz, Nicolaus Copernicus University in Torun, Poland

Adv Dermatol Allergol 2021; XXXVIII (4): 537-543

DOI: https://doi.org/10.5114/ada.2020.100747

\begin{abstract}
In most cases ecthyma gangrenosum is a consequence of Pseudomonas aeruginosa bloodstream infection in immunodeficient patients. This bacterium is characterized by multi-drug resistance and has a number of mechanisms that allow it to survive even in extreme conditions. The disease is characterized by an aggressive course involving the skin and mucous membranes, leading to ulceration with signs of necrosis within 12 to $24 \mathrm{~h}$. Treatment includes targeted antibiotic therapy and surgical cleansing of the wound. If the perianal area is occupied, a colostomy may be performed. Prevention of bacterial infections involves taking special precautions when handling a patient with immunodeficiency.
\end{abstract}

Key words: ecthyma gangrenosum, Pseudomonas aeruginosa, paediatric oncology departments.

\section{Introduction}

Ecthyma is one of the forms of lichen which involves dermis [1], whereas ecthyma gangrenosum is a pathognomonic disease that, in most cases, develops as a complication of Pseudomonas aeruginosa sepsis. It can also occur in previously healthy patients as a result of direct skin infection or secondary to bacteraemia caused by infection with other pathogens [2, 3]. It was first described in 1897 as bacterial vasculitis [3]. Speirs et al. in an article published in 1963 were the first to describe the correlations between agammaglobulinemia, sepsis, Pseudomonas aeruginosa and ecthyma gangrenosum [4, 5]. Most cases of this disease occur in South Asia [6].

\section{Characteristics of Pseudomonas aeruginosa}

Pseudomonas aeruginosa is a Gram-negative bacterium, whose genome is clearly larger compared to the other 25 most commonly sequenced bacteria. Knowledge of the genetic information of this microorganism, which consists of almost 6.3 million base pairs, gives a broader look at its role as a pathogen. Compared to other ge- nomes, it contains much more regulatory sequences, which allows it to adapt efficiently to environmental changes and survive under various conditions. About 150 genes are responsible for membrane proteins that are involved in the export of virulent agents outside the cell and the transport of antibiotics. In addition, they attach the structures responsible for mobility and adhesion. The active efflux system and low outer membrane permeability explain the resistance of the bacterium to first-line antibiotics [7].

This microorganism prefers aerobic conditions, but is also able to survive in an environment with limited oxygen or anaerobic conditions - in which case it breathes with nitrate or nitrite. Pseudomonas aeruginosa has a series of oxidases, which allow to lowering $\mathrm{O}_{2}$ levels, and thus create reduced-oxygen conditions, that are optimal for its growth. This microorganism is a saprophyte in high humidity environment and in the human intestine, it feeds by dead organic matter [8, 9]. Pseudomonas aeruginosa is able to survive at extreme temperatures and shows weakened sensitivity to antiseptics [10]. Virulent agents secreted by the bacterium include alkaline

Address for correspondence: Patryk Kaczor, Faculty of Medicine, Ludwik Rydygier Collegium Medicum, Nicolaus Copernicus University, 9 M. Skłodowskiej-Curie St, 85-094 Bydgoszcz, Poland, phone: +48 5258540 15, e-mail: patryk.kaczor97@gmail.com Received: 17.09.2019, accepted: 11.04.2020. 
proteases, lipases, elastases and toxins [7]. They lead to disruption of host tissue integrity by destroying specific proteins - elastin, transferrins and collagen. This microorganism is able to synthesize and secrete pyocyanin a blue dye with oxidation-reduction potential, which is a toxin with harmful effects on host cell mechanisms [11, 12]. It is suggested that this bacterium, through the production of toxins, is able to cause transient neutropenia due to the reduction of neutrocyte quantity and inhibition of their migration to the site of infection $[1,13]$.

Of all bacteria, it is Pseudomonas aeruginosa that contains one of the most advanced chemoreceptor systems. It shows negative chemotaxis towards isothiocyanate and thiocyanate esters and positive chemotaxis to amino acids, sugars and inorganic phosphates. So far 26 DNA segments have been identified to be responsible of encoding proteins involved in these processes [7].

\section{Pathogenesis}

Pseudomonas aeruginosa is one of the most important opportunistic pathogens responsible for human infections. This bacterium naturally lives in animal and plant tissues, as well as in swamps, soil and marine coastal areas. It is characterized by unusual multi-drug resistance and insensitivity to disinfectants. It mainly causes respiratory pneumonia, sepsis in burn victims and urinary tract infections in catheterized patients. Due to the high resistance to antibiotics, these diseases are difficult to treat [7].

Biofilm formation by Pseudomonas aeruginosa is another reason for the severity of infections caused by that pathogen, especially in interventional wards. This process occurs by attachment of the bacterium to the host's body using flagella and fimbria and by producing special exopolysaccharide - alginate [14]. It is an important component of extracellular mucus, which can probably lead to attenuation of phagocytosis by neutrophils and host macrophages, and thus to reduction of bacterial biofilm exposure to destruction by leukocytes [15]. Pseudomonas aeruginosa produces specific elastases that allow bacteria to reach the subcutaneous tissues as a result of the destruction of the wall of blood vessels, and thus are responsible for skin necrosis. The ulceration is caused by the release of proteases and exotoxin A by the bacteria [16].

This microorganism is a particularly big difficulty in paediatric patients with leukaemia. The multi-drug resistance Pseudomonas aeruginosa significantly limits therapeutic options, making infections one of the main causes of death in paediatric oncology wards [17].

\section{Ecthyma gangrenosum}

Ecthyma gangrenosum is an aggressive, infectious disease of the skin and mucous membranes, which in rare cases can take the form of vasculitis. Its development most often occurs as a result of direct infection of the skin of patients with chronic or immunodeficiency diseases and in patients with sepsis, most often with the aetiology of Pseudomonas aeruginosa (EG develops in up to $30 \%$ of people with bacteraemia [16]), however, there are cases of ecthyma gangrenosum in patients with a properly functioning immune system [18-20].

Paediatric patients with axillary and perianal skin fissures predispose to infection by this bacterium. They are often a side effect of chemotherapy or can arise directly as a result of malnutrition in the process of cancer in children [21]. Patients hospitalized for a longer period of time have more Pseudomonas aeruginosa colonies on their skin. This microorganism in patients with impaired immunity enters the body through microfissures in the skin, resulting mainly from thermal damage, vascular and urinary catheters, and bedsores [16]. Ear canal or gastrointestinal tract can also be the entrance for the bacteria [6].

Pseudomonas aeruginosa infection is the most frequent predisposition to ecthyma gangrenosum. Cases of infections by other pathogens are also reported, including Staphylococcus aureus, Streptococcus pyogenes, Serratia marcescens, Neisseria meningitidis, Neisseria gonorrhoeae, Klebsiella pneumoniae, Chromobacterium violaceum, Escherichia coli, Citrobacter freundii and Aeromonas hydrophila. There are single cases of infections caused by fungi, including Candida albicans, Aspergillus fumigatus, Fusarium spp., Curvularia spp., Exserohilum spp., Rhizopus spp. or Mucor spp. Herpes simplex virus may be responsible for ecthyma gangrenosum-like lesions. Viral infections can lead to a temporary impairment of the host's immune system or directly weaken the gastrointestinal defence barrier, which increases the risk of secondary infection with Pseudomonas aeruginosa [1, 9, 10, 19, 22, 23].

\section{Symptoms}

The lesions initially appear as red, painless macules, most often in the lumbar region, perineum and buttocks (57\%), limbs (30\%), face (6\%), neck and torso (6\%), which may be accompanied by non-specific symptoms, including abdominal pain, diarrhoea, fever and flu-like symptoms $[4,6,9,18,24]$. Ecthyma gangrenosum can present as single or multiple lesions [19]. The skin lesions then gradually increase and become papulae. The next stage is the stage of the hemorrhagic blister, which over time breaks and transforms into a black eschar surrounded by erythema - the transformation from a papula into an eschar takes 12 to $24 \mathrm{~h}$. At the site of skin disruption, necrotic penetrating ulcers eventually appear [4, 18, 23].

Pulido et al. in an article published in 2012 describe the case of an 11-month-old girl, whose first symptoms appeared 3 days before admission to the hospital in the form of discomfort during diaper change, caused by 
swelling and slight redness of the right labia. The patient was feverish and had previously been diagnosed with acute lymphoblastic leukaemia along with neutropenia associated with chemotherapy. After admission to the ward, no other skin symptoms were found, and thus the decision to administer cefepime and gentamicin was made. On the second day of hospitalization, a round spot with a diameter of $8 \mathrm{~mm}$ with associated erythema and central necrosis was noticed on the inner surface of the right labia major. After consultation, due to the inability to collect a skin point biopsy, a surgical biopsy was decided. During the operation, the lesion was removed completely and the samples were sent for microbiological tests, which confirmed Pseudomonas aeruginosa infection. The tissue around the wound was hardened, but according to previous suspicions, there were no purulent foci due to the patient's neutropenia $[19,25]$.

\section{Differentiation}

Pathologically changed areas of the skin have a characteristic morphology, therefore they should be quickly diagnosed in order to avoid serious complications [18]. In most cases, ecthyma gangrenosum in the form of necrotic ulcers surrounded by erythema is a pathognomonic symptom of Pseudomonas aeruginosa sepsis, most of the time in patients with impaired immunity and neutropenia. However, cases of infection with this pathogen without direct development of bacteraemia have been reported [26]. The increased risk of death in the event of incorrect identification of ecthyma gangrenosum may be due to the lack of sufficiently fast administration of appropriate antibiotics. Skin changes should be differentiated primarily from fungal infections, which often occur in patients with immunodeficiency [19]. The initial stage of the disease in the form of small papulae may be similar to chickenpox, but in the case of ecthyma gangrenosum they are rapidly increasing in size. Necrotic skin, in order to avoid administration of steroid drugs, should be differentiated from pyoderma gangrenosum, which is characterized by the absence of bacteria in the histopathological preparation and the presence of purulent lesions. Ecthyma gangrenosum, compared to usual cellulitis, has a faster course occurring with the characteristic evolution of the affected skin areas [2, 6].

Ishikawa et al. in their scientific work published in 2005 describe the case of a 4-year-old boy who, after antibiotic therapy (cefaclor, piperacillin, ampicillin + sulbactam), developed changes in the form of widespread necrotizing ulcers that showed similarity to ecthyma gangrenosum. Despite the lack of a positive result of microbiological examination, based on clinical symptoms, the patient was diagnosed with Streptococcus spp. group A infection. Histopathological examination should be performed in order to differentiate Pseudomonas aeruginosa infection from Staphylococcus spp. and Streptococcus spp. ones. In Pseudomonas aeruginosa case it is possible to observe a small inflammatory reaction, hemorrhagic necrosis with numerous eosinophilic bacteria in the surrounding of and directly in the adventitia and middle layer of blood vessels resulting in occlusive inflammation $[23,26]$, as well as the formation of a fibrin clot. On the other hand, in case of Staphylococcus spp. and Streptococcus spp. infections, a diffuse inflammatory reaction is observed, most of the time without the presence of bacterial cultures. However, the exact mechanism of ecthyma gangrenosum-like changes after antibiotic therapy remains unknown; the potential cause is the destruction of pathogens and follow-up release of toxins or the postantibiotic change in the intestinal microflora [27].

\section{Treatment}

In the case of ecthyma gangrenosum caused by Pseudomonas aeruginosa in patients with immunodeficiency, it is particularly important to make a rapid diagnosis of the causative agent and implement appropriate targeted antibiotic therapy $[10,21]$. Neutropenia in children may result in the rapid progression of the disease, and thus lead to necrosis of new tissues [18].

After confirming the diagnosis, targeted antibiotic therapy with the option of surgically resecting necrotic areas is currently preferred $[18,19]$. In addition, skin incision and tissue cleansing from necrosis are practiced, and in some cases skin transplantation is also considered $[21,28]$. It is suggested to use chlorhexidine and polymyxin B to wash the wound and its subsequent protection using paraffin dressings [10]. Due to the location of deep lesions in the dermis and high mortality, systemic treatment is preferred over surface therapy [1]. Until the results of microbiological tests are received, in patients with immunodeficiency and neutropenia with suspicion of ecthyma gangrenosum, empirical treatment with broad-spectrum $\beta$-lactam antibiotics is being implemented, including imipenem, aminoglycosides (gentamicin), cefepime and fluoroquinolones [19, 29]. Two of them should have proven effectiveness against Pseudomonas aeruginosa before. When the test results are obtained, the antibiotics should be changed to those with the narrowest spectrum, according to the antibiogram [19]. In oncological patients with Pseudomonas aeruginosa sepsis, the use of only one antibiotic is preferred; the effectiveness of combination therapy has not been proved. Prolonged infusion of antibiotics lasting several hours is in consideration; it has been proven in animal models that this strategy allows the minimum inhibitory concentration (MIC) to be maintained for a longer period of time, thus increasing the efficacy of therapy against Pseudomonas aeruginosa [29]. In 2002-2004, in Albany Medical Center in New York, USA, and in 2010-2011 in the Ohio State University Medical Center in Ohio, USA, single-centre observational studies were conducted on patients infected with Pseudomonas aeruginosa, which 
showed a reduction in mortality when using prolonged infusions of antibiotics [29-31].

Aitken et al. in a paper published in 2016 described the case of a 9-year-old boy with acute myeloid leukaemia relapse and neutropenic fever. They proved that the best therapeutic option for blood infection with the multi-resistant Pseudomonas aeruginosa, after colistin (which in patients with neutropenia has limited efficacy and the optimal dose is not known), is the ceftolozane/ tazobactam combination. The combination of ceftazidime/avibactam, which was effective in the treatment of multi-resistant Klebsiella pneumoniae sepsis, was also considered, but bacterial resistance and lack of efficacy were found in Pseudomonas aeruginosa infections. The final dose of the ceftolozane/tazobactam combination should, based on pharmacokinetic and pharmacodynamic analyses, be selected so that blood levels of a minimum of double MIC for ceftolozane are obtained throughout the whole treatment period. The antibiotic should be administered as a prolonged intravenous infusion lasting 3 h, given every 6 h. Such strategy allows maintaining a concentration of ceftolozane unbound to plasma proteins of a minimum of twice the MIC throughout the entire cycle of administration of one dose [17].

In paediatric patients who have had necrosis involving the anal sphincter in the perianal area, a temporary protective colostomy may be performed. This procedure allows to isolate locally altered, infected skin from the stool, and thus start the wound healing process. There are three types of colostomy: loop colostomy (most often performed for paediatric patients); end colostomy/single-barrel (Hartmann's operation - preferred in adults); and double barrel colostomy. A few months after completing the treatment of the perianal area, patients may undergo reversal colostomy and reconnection of intestinal loops to restore physiological intestinal continuity $[21,28]$.

Lau et al. in an article published in 2014 suggest that reducing the concentration of pyocyanin - a toxin produced by Pseudomonas aeruginosa - by neutralizing it as a result of antioxidant therapy or by completely inhibiting its synthesis may be an alternative, supporting method of treating infection caused by this pathogen [12]. If a fungal infection is suspected, systemic antifungal therapy should be implemented. In patients with immune disorders, including agammaglobulinemia, hypoglobulinemia or neutropenia, administration of immunoglobulins or granulocyte stimulating factors may be considered in order to increase the chances of recovery $[4,23]$. It is also necessary to provide adequate nutrition to the patient [16].

\section{Epidemiology}

Main risk factors for nosocomial infection with the multi-drug resistant Pseudomonas aeruginosa include pri- or administration of antibiotics, intubation and mechanical ventilation. However, up to $90 \%$ of blood infections occur through the central venous catheter. Ultimately, the development of bacteraemia significantly increases the mortality rate of nosocomial infections [14]. Cases of nonbacterial ecthyma gangrenosum have been reported in healthy individuals, without exposure to risk factors [18].

Pseudomonas aeruginosa is an opportunistic pathogen that is particularly common for skin, mucous membranes and subcutaneous tissue infections in immunocompromised and neutropenic children, most commonly as a result of acute leukaemia or other malignant neoplastic diseases, aplastic anaemia, hypogammaglobulinemia, agammaglobulinemia, steroid therapy or AIDS $[10,21,23]$. Reduced immunity, especially in patients from haematology and oncology departments who have undergone allogenic hematopoietic bone marrow transplantation, predispose to infection with Gram-negative bacteria, including the multi-drug resistant Pseudomonas aeruginosa [32]. Wounds in paediatric patients with immunosuppression and neutropenia are often infected by this pathogen [21].

This bacterium has the ability to create a biofilm, especially in environments with high humidity. Good conditions for this process are provided by hospital sanitary and water supply systems, which means that they can be a source of infection with this microorganism [32].

Depending on the source, the mortality of ecthyma gangrenosum with bacteraemia, especially caused by Pseudomonas aeruginosa infection, is $38-77 \%$, while in the absence of sepsis, this rate drops to $15 \%[18,23]$. A single skin lesion is associated with a better prognosis than multiple lesions [19]. In addition, septic shock, multi-drug resistant microorganism infection, neutropenia and delayed antibiotic therapy are associated with an increased risk of death [23].

\section{Resistance of Pseudomonas aeruginosa}

Pseudomonas aeruginosa is a multi-drug resistant pathogen against which most antibiotics are not effective [33]. Colistin, as a drug of last resort in case of patients with neutropenia, has limited effectiveness and the optimal dose is unknown [17]. The bacterium is characterized by overproduction of AmpC chromosomal cephalosporinases, which is probably associated with $\beta$-lactam resistance [33]. According to the scientific work of Rossi Gonçalves et al. published in 2016, carbapenems and $\beta$-lactams are the most commonly used antibiotics in Brazilian hospitals. $60 \%$ of Pseudomonas aeruginosa infections in these medical centres cannot be treated with them because of resistance to these drugs caused by producing specific metal- $\beta$-lactamases ( $\mathrm{MBL}$ - mainly: SPM-1, VIM, IMP-1) by these bacteria. Not all carbapenem-resistant strains produced $M B L$ - another possible explanation for bacterial resistance to this group of antibiotics is reduced expression of OprD porins [14, 33]. 
A characteristic feature of Pseudomonas aeruginosa is the extraordinary ability to develop resistance to further antibiotics. Beta-lactam antibiotics used to treat febrile neutropenia are not effective against many strains of this bacterium. In 2009-2012 in Italy, a total of 66 Pseudomonas aeruginosa-positive blood samples were taken from 9 haematological departments, followed by antibiograms; the results of this study are presented in Table $1[29,34]$.

The results of these studies explain the lack of effectiveness of empirical antibiotic therapy with $\beta$-lactams in the hospitals involved in the analysis, and thus the increase in the mortality of patients with neutropenia who developed Gram-negative bacteria bloodstream infection [29].

The problem of Pseudomonas aeruginosa drug resistance is also becoming significant in other countries. In 2009-2012, studies on 279 blood samples reported to the CDC (Centers for Disease Control and Prevention) and drawn from central venous catheters of oncological patients infected with this pathogen were carried out. Resistance to carbapenems and cephalosporins was found in $20 \%$ and in $14 \%$ of cases, respectively $[29,35]$.

\section{Prevention}

In the prevention of bacterial infections, including the multi-drug resistant Pseudomonas aeruginosa (MDRP), it is important to take special precautions which, when used simultaneously, significantly contribute to increasing the effectiveness of prevention against infections. These include: careful hygiene and hand disinfection; adherence to the principles of EBM (evidence-based medicine) regarding vascular catheters; placing patients (if possible) in isolated rooms; maintaining a clean hospital environment; implementation of special precautions against patients colonized with multi-drug resistant strains of bacteria. It is difficult to prioritize these activities; it is suggested to follow them simultaneously. In oncology departments, it is suggested to limit the use of broad-spectrum antibiotics when there is no need to ad-
Table 1. Results of Pseudomonas aeruginosa resistance to $\beta$-lactams in 9 haematology departments in Italy in 2009-2012 [29, 34]

\begin{tabular}{lc}
\hline Antibiotic & $\begin{array}{c}\text { Resistance number } \\
\text { of blood samples: } 66\end{array}$ \\
\hline Meropenem & $71 \%$ \\
\hline Ceftazidime & $55 \%$ \\
\hline Piperacillin + tazobactam & $42 \%$ \\
\hline
\end{tabular}

minister them as they are a risk factor for infection with multi-resistant strains [29]. In order to protect healthy children from infection, inflatable garden swimming pools should be regularly chlorinated since they can be a source of Pseudomonas aeruginosa infection [3].

In 2011-2014 at the University Children's Hospital in Muenster in Germany, the transplant department was renovated, with the main goal of reducing the environmental risk of infection with the multi-drug resistant strains of Pseudomonas aeruginosa. It was supposed to be achieved through creating adverse conditions for biofilm formation by these bacteria. New, specially designed, easy to disinfect shower drains have been implemented. Taps and shower heads were equipped with water filters and disinfecting siphons were installed under the sinks. The toilet bowls were replaced with rimless ones and a disinfectant was added to the flushing water. Patients' rooms were cleaned and disinfected on a daily basis and, at least once-a-month, environmental sampling for microbiological testing was started. In addition, hospital cleaning staff and other employees who have direct or indirect contact with patients have been thoroughly trained in hygiene procedures. All these actions eventually led to positive epidemiological changes presented in Table 2 [32].

\section{Summary}

In most cases, ecthyma gangrenosum is a complication of Pseudomonas aeruginosa sepsis. Most often it de-

Table 2. Epidemiological changes after renovation at the Children's University Hospital in Muenster, Germany in 2011-2014 [32]

\begin{tabular}{lcc}
\hline Factor & & Year \\
\cline { 2 - 3 } & 2012-2013 & 2014 \\
(before renovation) & 338 & 179 \\
\hline $\begin{array}{l}\text { Number of patients tested } \\
\text { with MDRP }\end{array}$ & $31(10.5 \%)$ & 3 (1.68\%) \\
\hline Number (percentage) of patients infected with MDRP & $13(3.85 \%)$ & $1(0.56 \%)$ \\
\hline Number (percentage) of deaths due to MDRP infection & $12(3.55 \%)$ & $0(0 \%)$ \\
\hline Number (percentage) of MDRP in toilet samples & 125 of $660(18.94 \%)$ & 23 of 275 (6.13\%) \\
\hline Number (percentage) of MDRP in shower drain samples & 52 of $641(8.11 \%)$ & 11 of 372 (2.96\%) \\
\hline
\end{tabular}

MDRP - multi-drug resistant Pseudomonas aeruginosa. 
velops in patients with immunodeficiency, but it can also occur in healthy people. Single or multiple lesions mainly involve the perineum, buttocks and lumbar region and progress rapidly; initially they appear as papules, which after 12 to $24 \mathrm{~h}$ eventually turn into a black scab with penetrating ulceration and necrosis. Empirical antibiotic therapy should be implemented as soon as possible and be changed to targeted one after obtaining the results of microbiological tests; Pseudomonas aeruginosa is an opportunistic multi-drug resistant pathogen, against which most antibiotics are not effective. Surgical cleansing of necrotic tissues and, ultimately, skin transplantation are also practiced. If the perianal area is affected with anal sphincter necrosis, a protective colostomy is performed, which is reversed after a few months. Prevention of infections with this pathogen primarily includes the use of special precautions by medical staff when dealing with a paediatric immunocompromised patient.

\section{Conflict of interest}

The authors declare no conflict of interest.

\section{References}

1. Zomorrodi A, Wald ER. Ecthyma gangrenosum: considerations in a previously healthy child. Pediatr Infect Dis J 2002; 21: 1161-4.

2. Koo SH, Lee JH, Shin H. Ecthyma gangrenosum in a previously healthy infant. Arch Plast Surg 2012; 39: 673-5.

3. Mull CC, Scarfone RJ, Conway D. Ecthyma gangrenosum as a manifestation of pseudomonas sepsis in a previously healthy child. Ann Emerg Med 2000; 36: 383-7.

4. de Almeida JFL, Sztajnbok J, Troster EJ, Vaz FAC. Pseudomonas aeruginosa septic shock associated with ecthyma gangrenosum. Rev Inst Med Trop Sao Paulo 2002; 44: 167-9.

5. Speirs C, Selwyn S, Nicholson D. Hypogammaglobulinaemia presenting as Pseudomonas septicaemia. Lancet 1963; 2 : 710-2.

6. Goolamali SI, Fogo A, Killian L, et al. Ecthyma gangrenosum: an important feature of pseudomonal sepsis in a previously well child. Clin Exp Dermatol 2009; 34: e180-2.

7. Stover CK, Pham XQ, Erwin AL, et al. Complete genome sequence of Pseudomonas aeruginosa PAO1, an opportunistic pathogen. Nature 2000; 406: 959-64.

8. Schobert M, Jahn D Anaerobic physiology of Pseudomonas aeruginosa in the cystic fibrosis lung. Int I Med Microbiol 2010; 300: 549-56.

9. Boisseau AM, Sarlangue J, Perel Y, et al. Perineal ecthyma gangrenosum in infancy and early childhood: septicemic and nonsepticemic forms. J Am Acad Dermatol 1992; 27: 415-8.

10. Chan YH, Chong CY, Puthucheary J, Loh TF. Ecthyma gangrenosum: a manifestation of Pseudomonas sepsis in three paediatric patients. Singapore Med J 2006; 47: 1080.

11. Prithiviraj B, Bais HP, Weir T, et al. Down regulation of virulence factors of Pseudomonas aeruginosa by salicylic acid attenuates its virulence on Arabidopsis thaliana and Caenorhabditis elegans. Infect Immun 2005; 73: 5319-28.
12. Lau GW, Hassett DJ, Ran H, Kong F. The role of pyocyanin in Pseudomonas aeruginosa infection. Trends Mol Med 2004; 10: 599-606.

13. Chusid MJ, Hillmann SM. Community-acquired Pseudomonas sepsis in previously healthy infants. Pediatr Infect Dis J 1987; 6: 681-4.

14. Rossi Gonçalves I, Dantas RCC, Ferreira ML, et al. Carbapenem-resistant Pseudomonas aeruginosa: association with virulence genes and biofilm formation. Brazilian J Microbiol 2017; 48: 211-7.

15. Leid JG, Willson CJ, Shirtliff ME, et al. The exopolysaccharide alginate protects Pseudomonas aeruginosa biofilm bacteria from IFN-gamma-mediated macrophage killing. I Immunol 2005; 175: 7512-8.

16. Kryeziu E, Kryeziu K, Bajraktari G, et al. Ecthyma gangrenosum in a patient with acute leukemia. Med Arh 2010; 64: 8-9.

17. Aitken SL, Kontoyiannis DP, Depombo AM, et al. Use of ceftolozane/tazobactam in the treatment of multidrugresistant pseudomonas aeruginosa bloodstream infection in a pediatric leukemia patient. Pediatr Infect Dis J 2016; 35: 1040-2.

18. Sarsu SB, Parmaksız ME, Yaman A. Ecthyma gangrenosum in a pediatric patient and review of the literature. Med Sci Discov 2016; 3: 200.

19. Pulido J, McMahon P, Treat JR, et al. Labial ecthyma gangrenosum in an immunocompromised infant with leukemia: heightening awareness for the urologist. Urology 2012; 80: 1366-8.

20. Clinic M, Clinic M. Ecthyma gangrenosum and multiple nodules: cutaneous manifestations of pseudomonas aeruginosa sepsis in a previously healthy infant. Pediatr Dermatol 2011; 28: 204-5.

21. Vuille-Dit-bille RN, Berger C, Meuli M, Grotzer MA. Colostomy for Perianal sepsis with ecthyma gangrenosum in immunocompromised children. J Pediatr Hematol Oncol 2016; 38: 53-7.

22. Reich HL, Williams Fadeyi D, Naik NS, Honig PJ. Nonpseudomonal ecthyma gangrenosum. J Am acad dermatol 2004; 50: 114-7.

23. Weiel JJ, Zhang CZ, Smith JA, et al. Ecthyma gangrenosum in pediatric patients: a three case series and review of the literature. J Clin Anat Pathol 2013; 1: 1-5.

24. Fergie JE, Patrick CC, Lott L. Pseudomonas aeruginosa cellulitis and ecthyma gangrenosum in immunocompromised children. Pediatr Infect Dis J 1991; 10: 496-500.

25. Singh N, Devi M, Devi S. Ecthyma gangrenosum: a rare cutaneous manifestation caused by Pseudomonas aeruginosa without bacteremia in a leukemic patient. Indian I Dermatol Venereol Leprol 2005; 71: 128-9.

26. Song WK, Kim YC, Park HJ, Cinn YW. Ecthyma gangrenosum without bacteraemia in a leukaemic patient. Clin Exp Dermatol 2001; 26: 395-7.

27. Ishikawa T, Sakurai Y, Tanaka M, et al. Ecthyma gangrenosum-like lesions in a healthy child after infection treated with antibiotics. Pediatr Dermatol 2005; 22: 453-6.

28. Khalil BA, Baillie CT, Kenny SE, et al. Surgical strategies in the management of ecthyma gangrenosum in paediatric oncology patients. Pediatr surg Int 2008; 24: 793-7.

29. Satlin MJ, Walsh TJ. Multidrug-resistant Enterobacteriaceae, Pseudomonas aeruginosa, and vancomycin-resistant Enterococcus: three major threats to hematopoietic stem cell 
transplant recipients. Transpl Infect Dis 2017; 19: 10.1111/ tid.12762.

30. Lodise TP Jr, Lomaestro B, Drusano GL. Piperacillin-tazobactam for Pseudomonas aeruginosa infection: clinical implications of an extended-infusion dosing strategy. Clin Infect Dis 2007; 44: 357-63.

31. Bauer KA, West JE, O'Brien JM, Goff DA. Extended-infusion cefepime reduces mortality in patients with Pseudomonas aeruginosa infections. Antimicrob Agents Chemother 2013; 57: 2907-12.

32. Kossow A, Kampmeier S, Willems S, et al. Control of multidrug-resistant Pseudomonas aeruginosa in allogeneic hematopoietic stem cell transplant recipients by a novel bundle including remodeling of sanitary and water supply systems. Clin Infect Dis 2017; 65: 935-42.

33. Deplano A, Denis O, Poirel L, et al. Molecular characterization of an epidemic clone of panantibiotic-resistant Pseudomonas aeruginosa. J Clin Microbiol 2005; 43: 1198-204.

34. Trecarichi EM, Pagano L, Candoni A, et al. Current epidemiology and antimicrobial resistance data for bacterial bloodstream infections in patients with hematologic malignancies: an Italian multicentre prospective survey. Clin Microbiol Infect 2015; 21: 337-43.

35. See I, Freifeld AG, Magill SS. Causative organisms and associated antimicrobial resistance in healthcare-associated, central line-associated bloodstream infections from oncology settings, 2009-2012. Clin Infect Dis 2016; 62: 1203-9. 